\title{
Philosophiques
}

\section{Structure et méthode dans la philosophie du sens et de la valeur}

\author{
Anna-Maria Vassilie-Lemeny et Sorin Titus Vassilie-Lemeny
}

Volume 2, numéro 1, avril 1975

URI : https://id.erudit.org/iderudit/203023ar

DOI : https://doi.org/10.7202/203023ar

Aller au sommaire du numéro

Éditeur(s)

Société de philosophie du Québec

ISSN

0316-2923 (imprimé)

1492-1391 (numérique)

Découvrir la revue

Citer cet article

Vassilie-Lemeny, A.-M. \& Vassilie-Lemeny, S. (1975). Structure et méthode dans la philosophie du sens et de la valeur. Philosophiques, 2(1), 83-102.

https://doi.org/10.7202/203023ar d'utilisation que vous pouvez consulter en ligne.

https://apropos.erudit.org/fr/usagers/politique-dutilisation/ 


\title{
STRUCTURE ET MÉTHODE DANS LA PHILOSOPHIE DU SENS ET DE LA VALEUR *
}

\author{
par \\ Anna-Maria \& Sorin Titus \\ Vassilie-Lemeny
}

\section{LES TROIS RELATIONS FONDAMENTALES}

Toute recherche philosophique doit tenir compte de la différence entre une quaestio facti et une quaestio juris. Même si elle n'est pas expressément spécifiée, il faut que cette différence soit au moins sous-entendue. La quaestio facti est la question fondamentale dans le domaine propre à la science, qui s'interroge sur le fait-d'être de tout ce qui est réel. Le domaine scientifique a comme relation principale la causalité, car tout fait ou phénomène doit, pour la science, être l'effet d'une cause. Cependant, certaines branches scientifiques (qui toutes recherchent la réalité) vont au-delà de la stricte cause. Elles se posent des questions sur la fin de l'existence, ou sur l'intention existentielle. Ceci, bien entendu, n'est le cas que des sciences «de l'esprit». Pour les autres, c'est-à-dire pour les sciences «positives 》, l'intention ou la fin en vue de laquelle se produisent les phénomènes et ont lieu les faits naturels n'a aucune importance. La préoccupation intentionnelle apparaît en même temps que l'étude de la conscience humaine, l'intentionnalité étant un des mouvements et une des expressions les plus représentatives de celle-ci. Mais chacune des sciences de l'esprit accorde une fin à part à l'homme, donc chacune ne voit dans l'existant humain qu'un seul aspect de son être. Ceci ne serait en rien si grave ou nuisible, si elles se contentaient

* Par philosophie du sens et de la valeur, l'auteur entend une théorie synétique de la valeur considérée dans une perspective épistémologique. (Note de l'éditeur). 
de cela, mais elles veulent chacune imposer au sujet humain une fin en conformité avec l'objet de leurs recherches respectives, considérant cette fin comme ultime et complète pour l'être entier de l'homme. Pourtant, aucune de ces fins ne peut justifier une telle prétention, pour la simple raison que la fin, considérée comme telle, n'implique pas la justification. Pour qu'une justification soit possible, un sens est nécessaire. Nous allons voir bientôt que la distinction entre sens et fin est très importante si l'on veut comprendre la quaestio juris et son domaine. La confusion entre ces deux concepts, si largement répandue et si profondément ancrée dans la pensée, ne peut que nuire à la philosophie. Aussi, ce serait le moment de nous en débarrasser. Il est vrai que parfois la fin s'attache au sens, ce qui rend plus difficile leur séparation. Mais à un examen attentif, ce n'est pas une tâche trop ardue que de les disjoindre même dans ce mariage d'intérêt, où la fin est intéressée à obtenir une justification, qui ne peut être due quau sens.

Par ailleurs, nous ne pouvons pas non plus hiérarchiser les différentes fins établies par les sciences, vu que toute fin est indifférente à la valeur. On sait, sans doute, qu'une des premières conditions de la recherche scientifique est d'éviter strictement les jugements de valeur, considérés comme dépourvus d'objectivité. Or, l'objectivité pour la science, ce n'est que la considération de la réalité de facto, sans aucune préoccupation concernant le droit d'être, ou la valeur justificative de l'existence.

Donc, le sens d'une part, et d'autre part la valeur nous montrent la possibilité de dépasser les relations cause-effet et finmoyens. Ainsi se révèle l'ordre de la quaestio juris, où tout existe seulement dans la mesure où il peut légitimer son existence. Cela revient à dire que dans cet ordre rien ne saurait être sans avoir de la valeur par la signification, c'est-à-dire par l'expression du sens qu'il porte. Il est évident que la quaestio juris concerne principalement la cogitation. Bien plus encore, c'est la question fondamentale de la cogitation, quelle que soit la nuance de cette dernière: philosophique, mathématique, morale, ou artistique. En vérité, plus nous chercherions à réduire le sens de la cogitation, plus il ressortirait avec éclat, cogiter pouvant être défini seulement comme un contenu intelligible activé par une ten- 
dance. Mais si la valeur dépend du sens, la relation inverse est vraie aussi, car tout sens, pour exister comme tel, doit être valable, c'est-à-dire qu'il doit avoir valeur de vérité, faute de quoi il devient un non-sens.

Par conséquent, nous pouvons dire que le sens et la valeur sont des éléments corrélatifs, pouvant se substituer réciproquement, similaires aux bases «duales » de formes coordonnées des espaces vectoriels de la théorie des structures algébriques. En d'autres termes, dans la sphère de la cogitation, la quaestio juris s'impose comme question essentielle, constitutive, comme question déterminante des relations transcendantales de cogito-cogitatum, soit que la justification vienne de la part du sens, soit qu'elle s'obtienne grâce à la valeur. Cela veut dire que dans cette sphère aucune chose ne peut être que dans la mesure où elle prouve et met en évidence sa signification, ou sa validité.

Mais si la relation fin-moyens et encore moins celle de cause-effet ne peuvent satisfaire l'ordre de la quaestio juris, y a-t-il une autre relation qui soit à même de procurer la structure formelle de cet ordre? Certes, une telle relation apparaît nécessaire, vu que sans elle aucun acte de cogitation ne serait possible. Or, ce ne peut être que la relation de présupposition-implication, la seule capable d'ordonner les sens dans un continuum valorique. Pour comprendre la dialectique entre présupposition et implication, nous dirons que tout sens supérieur implique un sens inférieur, ou inversement, que ce dernier présuppose le premier. Par sens supérieur nous devons entendre le sens qui constitue un degré supérieur d'autonomie par rapport au sens inférieur. Le sens a de la valeur par sa puissance de signifier, et la signification s'évalue par son évidence ${ }^{1}$. Certes, toutes les significations ne sont pas également évidentes, mais être-évident ce n'est pas être tout simplement frappant. La valeur de la signification dépend du degré d'intériorité de l'évidence, car en d'autres termes, elle est directement proportionnelle à l'intensité de la puissance d'idéation du cogiter. On observe que toutes les notions synétiques ${ }^{2}$ (signification, évidence, puisssance d'idéation et, certes, sens) doi-

1. Voir à ce sujet Les éléments «duals》 et la dualité des principes, dans Sapienza, Napoli, no 2, 1974.

2. Terme provenant du grec « sunésis » qui signifie « sens». 
vent être considérées réflexivement, vu que ce sont des notions circulaires, aussi bien transitives qu'intransitives. C'est pour cette raison qu'elles sortent de la logique ordinaire (tel l'ensemble de tous les ensembles) ${ }^{3}$. Et c'est pourquoi aussi, dans tout sens nous distinguons, comme nous allons le faire voir, un contenu intelligible (entendu) porté par une tendance synétique vers un entendement ou intelligence d'un ordre supérieur.

Mais la possibilité qu'a l'évidence de s'intérioriser, c'est-àdire de s'approfondir par la puissance d'idéation, prouve la possibilité de l'autonomie synétique, dont elle est la conséquence. En effet, plus un sens est autonome, plus l'évidence de sa signification sera difficile à découvrir, en raison même de son intériorité. Mais elle sera à la fois plus certaine, car autonomie signifie indépendance et de cette manière assure la certitude. Par conséquent, le critère de la hiérarchie des sens est en somme donné par l'autonomie valorique, et cette autonomie attire après soi la finesse ainsi que la profondeur de l'évidence, dont jouit la signification.

Nous avons donc à retenir qu'il y a trois catégories de relations : la causalité, la finalité et l'implication. Les deux premières forment l'objet des recherches scientifiques, c'est-à-dire des sciences positives et des sciences «de l'esprit». La troisième, qui exprime l'activité synétique, représente la préoccupation exclusive de la philosophie, la seule discipline capable de demander à l'être une justification (un fondement) qui dépasse l'origine naturelle et le but réel de tout fait-d'être. C'est pourquoi, seule la philosophie a le droit (et, assurément, le pouvoir) de s'interroger sur la validité des êtres, d'apprécier leur valeur existentielle, et d'établir, selon le critère de l'ordre synétique, si une hiérarchie des valeurs est possible ou non. Sans doute, la philosophie tiendra compte des recherches scientifiques, et les utilisera pour ordonner les sens et élaborer les motivations dans la sphère de la réalité. Mais les relations causale et finale ne sont point suffisantes pour révéler ce que l'on doit entendre par une chose et quelle est sa signification. En vérité, la cause efficiente indique seulement la source de l'événement, son mode de prendre «naissance». Quant à la

3. Cf. Esquisse pour une théorie de la vérité, dans Archives de Philosophie, Cahier IV.1968. 
cause finale, elle ne montre que la tendance du fait ou du phénomène étudié, son déploiement et son devenir vers quelque chose qui peut être dépourvu de tout «entendu», car aucunement capable de se faire entendre. En tout cas, ce sont les recherches scientifiques elles-mêmes qui démontrent qu'autant la «source » que la «tendance»comme telles restent en dehors de l'activité synétique, du moment que la rigueur scientifique impose des limites strictes à ces recherches. Et nous avons déjà eu l'occasion de constater que ces limites sont fournies par la relation de causalité pour les sciences naturelles, et par celle de finalité pour les sciences spirituelles.

Il est, donc, évident que la valeur d'une chose ne saurait être, elle non plus, appréciée si l'on se fonde sur ces deux relations, vu qu'elle est un terme auquel nous ne pouvons aboutir que par le contenu intelligible de la chose respective, de même qu'en algèbre nous ne pouvons accéder à la structure que par la loi de composition qui la définit. Aussi la valeur est un élément qui transcende la science (soit naturelle, soit spirituelle), tout comme un acte généreux, lequel, en pénétrant la vérité morale, la rend claire et de la sorte transcende la justice, rigoureuse, mais aveugle.

Pour mieux comprendre le sens et mettre en relief son caractère spécifique, examinons-le une fois de plus en contraste avec la fin. Nous allons ainsi constater que cette différence peut être établie suivant plusieurs points :

1. La fin, étant un but, ne fait qu'indiquer la direction, tandis que le sens signifie, donc rend possible, suscite et détermine un entendement.

2. La fin n'appartient pas à la puissance d'idéation, vu qu'elle est constituée par la volonté pratique de réaliser quelque chose à l'aide de certains moyens. Sans doute, il se peut que parfois elle utilise des moyens provenant de l'intelligence, mais cette intelligence n'est ni nécessaire, ni suffisante. Et même dans le cas des instruments intelligents, le caractère fondamental de fin reste entièrement pratique. Ainsi, la finalité est en dehors de la stricte cogitation, de l'ordre théorique de la conscience, et partant elle n'a pas besoin d'être justifiée par cette cogitation, comme on le 
voit dans l'adage: «Hoc volo, sic jubeo, sit pro ratione voluntas». En somme, toute fin est, d'une manière plus ou moins manifeste, tributaire d'une des multiples formes de la volonté de puissance. Le sens, au contraire, est un acte d'idéation, et peut donc se justifier seul, sa justification étant étrangère à la volonté et à tout rapport de finalité.

3. Par suite, une fin ne saurait être significative que si elle accompagne un sens et profite de sa signification.

4. La fin est un terminus final, notamment : celui qui termine la relation de finalité ; il arrête la tendance intentionnelle, c'est-àdire qu'il clôt l'intention suivie par les moyens. Le sens marque un mouvement opposé, vu qu'il soutient en permanence la cogitation comme telle; autrement, tout acte du cogiter perd sa valeur. Si le sens ne signifiait pas un continuum infini, la cohérence de la cogitation ne serait pas possible. Ou, exprimé en termes plus formels, si l'activité synétique manquait d'établir un enchaînement par implication (ou, en direction inverse, par présupposition), une pensée conséquente ne pourrait parvenir qu'à des conclusions discrètes (discontinues). Donc, un sens dénué de continuité infinie est voué au non-sens, car la rupture de la cohésion synétique provoque la désagrégation du contenu intelligible, et partant le discrédit de la pensée même. Cette dernière a, en vérité, besoin d'une puissance d'unification permanente, afin de pouvoir se faire entendre.

Aussi, l'idée de sens doit-elle impliquer une liberté concrète (concret étant synonyme de continu), pour se charger elle-même de sens; c'est une liberté que toutes les propriétés synétiques exigent, faute de quoi, elles n'auraient pas de plénitude idéale. Quant aux motifs inclus par le sens, c'est-à-dire les facteurs qui le rendent évident, ils réclament aussi des possibilités intarissables, irréductibles, de même que, dans la série des puissances transfinies, aucun terme ne peut être réduit à un autre. Au contraire, les moyens se réduisent nécessairement à la fin, celle-ci fermant leur suite, comme réplique des motifs, auxquels le sens ouvre la perspective.

5. Le sens contient la fin, tandis que l'inverse n'est pas valable. A vrai dire, le sens n'a aucune fin, mais c'est la fin qui doit avoir 
du sens pour être, sinon elle ne saurait être intelligible, que l'on comprend aisément. Ou, exprimé en termes logiques: le sens entre dans la définition de la fin, mais pour définir le sens, l'intervention de la fin est superflue et même génératrice de confusions, ce qui ressort de toutes les différences remarquées aux points antérieurs.

\section{CRITÈRE DE L'UN ET DE LA CONTINUITÉ}

Les recherches menées jusqu'ici nous ont révélé que le sens offre deux aspects: l'un d'intelligibilité, l'autre de tendance. Ceci veut dire que le sens doit être considéré à la fois comme contenu intelligible, ou «ce que l'on entend par», que nous allons appeler l'entendu, et comme mouvement de ce contenu, c'est-à-dire comme tendance, vers une intelligence d'un ordre supérieur. Donc, cette intelligence est l'acte vers lequel tout entendu est en puissance ouvert; aussi n'est-elle pas autre chose que l'entendement. Sans doute, la distinction des deux aspects ne représente qu'un procédé analytique de recherche, une séparation méthodique en vue de découvrir le caractère propre, intime du sens. Il est évident que ni l'entendu, ou contenu intelligible, ni la tendance ne peuvent séparément constituer le sens, vu que ce dernier est $u n$ acte d'intelligence, un acte conduisant à la compréhension. Par conséquent, pour mettre en relief qu'il est la partie commune de ces deux aspects, nous allons l'exprimer par une intersection :

$$
\text { Sens }=\text { (entendu / tendance). }
$$

Cette expression nous rend la structure synétique. Mais nous accentuons de nouveau que nous présentons le sens comme une structure seulement pour des raisons méthodiques.

Allons voir maintenant s'il est possible de trouver un critère pour estimer la structure synétique. Tout ouvrage logique, mathématique, ou philosophique, ainsi que toute oeuvre d'art, peut être considéré comme un système de pensée. Certes, le système implique l'ordre, et nous sommes incapables de construire quoi que ce soit sans un certain ordre. Mais «ordre» pour la pensée signifie consécution synétique, ce qui veut implicitement dire constitution de rangs. Et cela n'est, en somme, qu'une activité de hiérarchisation des valeurs. Aussi ne saurait-on penser, du 
moins philosophiquement, sans effectuer une évaluation. C'est pourquoi il est si important de trouver, pour une telle évaluation, une méthode.

Mais, tout comme pour construire un système on a besoin d'un certain nombre d'axiomes, pour élaborer une méthode d'évaluation plusieurs idées principales sont nécessaires. Ces idées constituent un ensemble fondamental que nous allons utiliser comme étalon, car il nous faut certains points de repère pour être capable d'apprécier les graduations de la valeur. Essayons donc d'établir quels sont les éléments de cet ensemble, en ne perdant pas de vue que le système estimé aura d'autant plus de valeur qu'il réunira des propriétés conformes à ces éléments-étalon.

L'unité est la possibilité d'un ensemble d'entités individuelles d'être représenté comme un seul individu, de même qu'un nombre cardinal transfini représente un ensemble infini de nombres; la cohérence de ces nombres, ou, plus exactement, la continuité de l'acte de dénombrer assure leur unité.

L'univocité signifie la possibilité d'attribuer un seul sens à un ensemble de sens, tel un cycle-limite de la théorie qualitative des équations différentielles, c'est-à-dire une courbe intégrale fermée, sur laquelle se déploient d'autres courbes intégrales sous forme de spirale.

L'unicité est la possibilité qu'un seul élément ne puisse être représenté par aucun autre élément, de même qu'un anneau commutatif ne peut avoir qu'un seul élément-unité, irremplaçable par aucun autre élément. Nous pouvons aussi définir l'unicité comme l'impossibilité d'être déduit de ou réduit à tout autre chose $^{4}$. Donc l'unicité est la puissance de se fonder soi-même, et par suite d'être indépendant.

L'universalité signifie la possibilité d'inclure tout acte synétique, pareille à un idéal maximal pour les idéaux qu'il inclut (selon le théorème de Krull). En d'autres termes, l'universalité est la puissance d'impliquer tout ce qui peut satisfaire les éléments-étalon précédents. Ainsi, « universel » est ce qui subsiste

4. Cf. Les éléments «duals» et la dualité des principes, dans Sapienza, Napoli, no 2, 1974. 
aux implications synétiques, étant le présuppposé de toute activité intelligible. C'est pourquoi, par universalité, nous devons entendre non seulement une capacité d'être totalement inclusif, mais aussi une faculté de constituer un modèle qui fonde et édifie, un archétype.

On observe que ces quatre éléments ont tous quelque chose de commun : ils sont des dérivés de l'idée de l'Un, et comme tels ils représentent les parties analytiques d'un seul acte de synthèse appelé unification. Donc, étant des composés de la forme simple de l'Un, nous allons les nommer attributs de l'Un, ou attributs valoriques.

L'unilatéralité n'est pas un attribut de l'Un, mais son mode de manifestation dans la réalité. A vrai dire, l'Un ne peut être connu qu'unilatéralement, car il est indivisible et par suite n'offre à connaître qu'un seul aspect. Mais cet aspect est son être même, l'unicité latérale découlant de l'universalité de son être.

Ainsi, les attributs de l'Un peuvent être considérés comme points de repère pour estimer la puissance d'entendre, car ils déterminent une voie qui assure l'unification du contenu intelligible. Faute d'une telle unification, il est évident que toute intelligibilité serait compromise. En vérité, «J'entends», c'est-à-dire l'acte par lequel le Moi se montre capable d'entendement (d'intellection), est lui-même un acte unificateur de la conscience. D'ailleurs, sur cet acte et son importance nous reviendrons plus loin, au paragraphe suivant. Pour le moment, il suffit de retenir que l'intelligence, ou cornpréhension de toute chose présuppose nécessairement une puissance unificatrice de la conscience spirituelle: c'est la puissance d'idéation.

Cependant, nous avons constaté que le contenu intelligible, nommé entendu, n'est qu'une manière incomplète d'envisager le sens. A vrai dire, l'entendu doit être doublé par la tendance, afin de signifier authentiquement le sens. Certes, l'entendu comme tel n'est à même ni d'expliquer le mouvement dans une direction, ri de justifier l'activité de conduire vers une destination, mouvement. et activité que le sens implique en une égale mesure. Donc, nous devrons à présent concentrer notre attention sur la tendance, pour 
rendre l'intégrale synétique, c'est-à-dire l'entière structure analytique du sens. Nous allons remarquer, d'après la manière dont la tendance fait corps commun avec l'entendu, qu'une telle structure est en vérité un seul acte. Et dans cet acte, comme nous l'avons déjà dit, si l'on distingue deux aspects c'est seulement pour mieux l'expliquer.

Nous avons constaté que plus le contenu intelligible d'un système jouit des attributs de l'Un, plus le système a de la valeur. Mais comment procède-t-on quand on doit comparer et évaluer deux ou plusieurs systèmes qui sont également capables de ces attributs valoriques? Comment pouvons-nous les ordonner et hiérarchiser suivant leur propre valeur, quand chaque attribut est lui-même gradué? Ainsi, est-il évident qu'un système peut être plus ou moins unitaire, univoque, unique et universel. Dans ce cas, la décision appartient à la tendance, qui a une certaine intensité, due à l'impulsion qui l'anime. A vrai dire, les attributs valoriques représentent des actes de cogitation, et par suite il ne convient pas de les considérer quantitativement. Une appréciation quantitative de ces actes signifierait leur décomposition en parties et l'estimation des parties séparément, ce qui serait une grosse erreur, comme l'a si brillamment montré Bergson. Par conséquent, la seule détermination possible est celle qui provient de l'intensité, qui elle nous donne l'acte entier. L'intensité révèle l'acte non pas comme un objet abstrait, mais dans son activité même. Alors, en tenant compte que l'intensité amène à la lumière le caractère propre, spécifique de la tendance, si nous parvenons à l'évaluer, cela signifie que nous avons trouvé la valeur complète du sens.

Donc, pour estimer l'intensité et par là définir la tendance, nous déterminerons les attributs qui représentent d'une manière analytique la continuité, vu que la puissance ou l'impulsion qui génère la tendance est un continuum, ne pouvant être conçue autrement qu'en tant que source inépuisable. Ainsi, serons-nous amenés à établir de nouveau quatre attributs, à savoir : la convergence, la conséquence, la cohérence et la cohésion, que la Continuité synthétise en un tout, en leur donnant une forme simple qui les transcende. Il est évident que cette forme ne peut être que la forme simple de l'Un. 
Passons maintenant à la définition de ces attributs ${ }^{5}$.

La convergence signifie l'intention vers le même but de plusieurs tendances disparates, c'est-à-dire la direction des tendances de telle sorte qu'elles visent un idéal commun. Donc, pour être convergent il faut avoir comme «spiritus rector 》 la même intention, tout comme l'infinité possible des nombres entre deux nombres réels intentionne vers la puissance infinie-actuelle du continuum.

La conséquence signifie l'observance et la conservation d'un lien entre les diverses tendances, donc la permanence d'une connexion plus ou moins étroite. En d'autres termes, être conséquent veut dire suivre un certain ordre des actes, dont la consécution s'accomplit par une relation implicative, ayant un certain degré de rigueur, telle une série de morphismes qui se succèdent en s'impliquant, pour structurer une catégorie algébrique.

La cohérence veut dire l'interpénétration plus rigoureuse des parties consécutives, si bien que chaque partie soit la conséquence d'une autre partie (pas nécessairement précédente). Ainsi, une chose est cohérente si elle possède une détermination exacte par rapport aux termes avec lesquels elle se trouve en relation, et de plus si l'exactitude est due à l'observance d'un principe majeur présupposé. Nous trouvons une telle exactitude déterminative, génératrice de cohérence, dans tous les raisonnements mathématiques.

La cohésion signifie la conformation et la contenance en un seul tout ou entier, structuré simplement dans sa totalité, par suite de la parfaite conjonction relationnelle. Cet entier existe de façon intégrale comme un individu, constitué de manière continue ( sans aucun saut, ni lacune). Avoir de la cohésion veut dire être construit par des relations unificatrices strictes, donc édifier une seule structure rigoureusement inclusive, contenant ses éléments et formant corps commun avec eux (corps dans le sens de participation). Aussi, cette structure est-elle réfractaire à toute division, comme un idéal maximal qui inclut tous les idéaux d'un anneau local, sans être divisé par eux.

5. Toutes les définitions mathématiques ont été données dans Esquisse d'une théorie arithmétique du sens et de la valeur (en roumain), publié dans Études et recherches mathématiques, Bucarest, no 6, 1968. 
Il est évident que la relation qui ordonne ces quatre attributs, à l'aide desquels nous évaluons l'intensité, c'est l'implication. En effet, chaque attribut implique toutes les propriétés des attributs précédents, ayant au surplus d'autres caractères propres par lesquels il dépasse ses prédécesseurs. Cette connexion implicative rend possible l'ordre croissant vers une unification toujours plus rigoureuse, que nous remarquons dans la série des quatre termes examinés. Par suite, on comprend sans difficulté qu'un tel mouvement unificateur doit aboutir à la forme simple du continuum. Aussi peut-on considérer ces termes comme des fonctions de la Continuité, sans oublier, toutefois, qu'ici nous avons affaire à une méthode, qui ne peut être qu'analytique.

Ainsi, nous espérons avoir montré qu'en estimant la tendance à l'aide de l'ensemble de la Continuité, on précise l'estimation de l'entendu, effectuée au moyen de l'ensemble de l'Un. De cette façon il est possible de trouver la valeur de l'entière structure synétique. Certes, les limites que tout article doit respecter nous empêchent d'entreprendre une analyse plus détaillée de l'intersection des deux ensembles. C'est une question que nous nous proposons de traiter dans un essai à part.

\section{CRITÈRE DE L'UN ET DU MULTIPLE}

Donc, nous avons constaté que le sens a deux caractères fondamentaux: l'intelligibilité et l'orientation. De même, notre recherche nous a montré que la valeur est fonction de l'intensité du contenu intelligible. Mais cette intensité est possible seulement si le sens s'oriente dans une certaine direction. En d'autres termes, la tendance doit suivre un certain mouvement, afin que l'intensité puisse compter comme facteur d'évaluation. Nous avons vu que ce mouvement en est un de concentration, assurant ainsi la continuité de la tendance. Cela nous permet d'établir une correspondence biunivoque entre les deux ensembles d'attributs ( $U$ et $C$ ), et de rendre la valeur de la structure synétique parfaite. Cette structure représente, en vérité, tout sens appartenant à la cogitation comme telle, c'est-à-dire à l'activité spirituelle pour laquelle la stricte puissance d'idéation suffit. A cet égard, nous avons l'exemple éloquent des mathématiques, vu que dans leur domaine rien ne saurait être de facto sans être en même temps (ou plu- 
tôt au préalable) de jure. Et il n'y a point de relation mathématique qui ne se fonde toujours sur une relation mathématique. Il en résulte, donc, que la quaestio facti peut coïncider avec la quaestio juris ; mais pour que cela soit possible, le domaine de coïncidence doit être régie par le sens et la valeur seuls.

Cependant, nous savons fort bien qu’à côté des mathématiques pures il y a aussi les mathématiques appliquées dans un très vaste domaine, étranger à tout ordre idéal. Cette constatation nous permet d'inférer qu'en plus de l'activité synétique stricte, pour laquelle sens et valeur constituent l'ontologie même, on peut concevoir un ordre synétique appliqué à un domaine différent : la réalité. Cet ordre s'applique dans la mesure où il révèle la signification du réel. Mais il est évident que dans ce cas la structure synétique ne peut plus être celle que nous avons rencontrée jusqu'ici, car maintenant il s'agit d'un être qui existe indépendamment du sens dont il est porteur. En somme, dans la réalité aucun être n'a besoin de sens, non plus que de valeur, pour qu'il soit réel; le seul fait lui suffit afin d'être comme tel. Les sciences sont les premières à le prouver, du moment qu'elles étudient ce qui est strictement de facto, en évitant par principe toute préoccupation justificative des phénomènes réels. Suivant nos recherches précédentes, la justification est due soit au sens, soit à la valeur. Certes, nous avons ici encore une preuve marquant la distinction entre les sciences, d'une part, et des disciplines comme: les mathématiques, l'art, la philosophie, la religion, d'autre part. Tous les éléments et les structures de ces disciplines ont leur fondement dans le même couple: (sens, valeur), nécessaire et suffisant pour l'existence.

Donc, la question qui s'impose à présent est celle de savoir en quoi consiste la structure synétique et comment se fait l'évaluation d'un être pour lequel le sens et la valeur ne sont pas essentiels. Il est vrai que l'aspect intelligible d'un tel être doit rester identique à celui du sens strict, car, ainsi que nous l'avons déjà montré et nous y reviendrons bientôt, nous sommes incapables d'entendre quoi que ce soit sans une activité unificatrice de l'esprit. Dès lors, ce sont toujours les attributs de l'Un, examinés plus haut, qui nous permettent d'estimer l'entendu ou contenu intelligible de l'être réel. Mais la situation n'est plus la même 
quand on arrive à la tendance. Pour reconnaître la direction suivie par cette dernière, nous n'avons qu'à suivre le développement ontologique du réel. En analysant donc la réalité dans son mode d'être naturel, nous remarquons dès le commencement que c'est un composé variable, vu qu'aucun de ses éléments ne se trouve être libre, c'est-à-dire pur et simple, mais peut exister seulement dans des compositions. Cette remarque initiale de la complexité s'affermit à mesure que nos recherches découvrent l'orientation synétique propre dans ce domaine. Ainsi, du fait que la réalité est complexe et variable, nous pouvons inférer qu'elle se développe par multiplication. En effet, le monde réel ne subsiste qu'en tant qu'il se multiplie. Pour couvrir l'entière activité de multiplication, nous avons à découvrir tous les actes multiplicatifs qui la composent. Aussi allons-nous procéder, une fois de plus, d'une manière analytique pour déterminer et formuler la tendance, dont l'intersection avec l'entendu nous permettra d'évaluer la structure synétique de la réalité.

Mais il n'est pas difficile d'observer que la multiplication consiste en quatre fonctions analytiques, formant une série ordonnée par implication croissante. Essayons de les expliquer en quelques mots.

La multitude signifie quelque chose de composé ou de complexe, ce qui dans le monde réel est non seulement un état de fait, mais aussi un caractère synétique. Sans doute, la multitude représente le premier signe de la direction dans laquelle la réalité tend, vu que c'est la fonction la plus élémentaire et commune, étant la propriété la plus répandue parmi les êtres réels. Mais c'est pour cette raison même que sa puissance de signification est la moindre.

La multiplicité signifie un accroissement synétique, et de cette façon pousse la tendance vers une valeur plus haute, car les chances de se perpétuer dans la réalité augmentent proportionnellement à l'impulsion multiplicative. On sait très bien que les plantes et les animaux défendent leurs espèces principalement par leur puissance de se multiplier. Cette puissance est, en effet, la dernière arme pour sauver la multitude. En vue de chaque être réel, l'activité de multiplication est une des plus précieuses. 
La valeur de cette activité est d'autant plus grande, que l'être est moins développé.

Par conséquent, la multiplicité assure la persistance des espèces. Toutefois, cette persistance ne peut être stable; elle existe comme perpétuelle transformation par des actes destructifs et ré-générateurs. Ainsi, la réalité est dans une fluctuation ininterrompue, grâce à la diversité des voies et à la variété des relations employées. Dans ce domaine rien n'est fixe et ankylosé, car être-réel signifie être-changeant, soit comme développement, soit comme destruction de l'être. Tout développement est, néanmoins, impossible sans une activité multiforme, vu que plus un individu agit en des formes multiples et diversifiées, plus son rang dans la hiérarchie des êtres est élevé. Donc, par multiformité nous devons entendre un repère synétique, marquant encore un degré que la tendance peut atteindre dans le monde réel.

Nous arrivons, enfin, à la dernière et, du point de vue valorique, à la plus importante des fonctions de la série: la multilatéralité. Son importance provient, en effet, de l'actualisation de la tendance en de nombreuses directions et de l'extension des structures ontiques, ramifiées pour embrasser le plus possible du domaine réel. Certaines de ces directions peuvent conduire vers des fins contradictoires, vu que la multilatéralité implique toutes sortes d'impulsions, capables d'arriver finalement à s'opposer et à se combattre réciproquement. Certes, le mouvement multilatéral tend à embrasser tout, mais il cherche à satisfaire cette tendance par un maximum de multiplication. En d'autres termes, ce mouvement s'efforce de comprendre l'entier, mais ne réussit qu'une extension plus ou moins large, car son processus est d'amasser une multitude de parties. Or, quel que soit le nombre des parties amassées, il ne peut signifier un entier, qui doit être conçu comme un ensemble ordonné (système). Aussi, avons-nous plus haut considéré l'entier comme un univers, et l'universalité comme capable de comprendre le système. A vrai dire, l'universalité procède par inclusion, et c'est par là qu'elle diffère totalement de la multilatéralité. D'ailleurs, cette manière de s'étendre pour couvrir l'entier à l'aide des parties est prouvée par les équations aux dérivées partielles, qui trouvent 
les plus vastes applications dans les phénomènes de la nature, et constituent pour cette raison la théorie la plus importante de la physique-mathématique. On sait que dans cette théorie l'intégration se fait seulement au moyen des dérivées partielles, c'està-dire en totalisant les parties en vue d'obtenir l'entier. Sans doute, nous avons affaire ici plutôt à un développement, qu’à une inclusion authentique de l'entier.

Nous espérons avoir montré ainsi que les quatre fonctions : multitude, multiplicité, multiformité et multilatéralité sont disposées dans un ordre valorique croissant. En effet, l'homme ne représente-t-il pas le maximum de valeur dans le monde réel justement parce qu'il est multilatéral, étant capable du plus grand nombre de directions d'activité ? Mais on peut facilement observer que ces quatre fonctions sont les dérivées ou indices par lesquels un principe opère, de telle sorte qu'il dirige l'estimation de toute la réalité. Il est évident que ce principe ne peut être que le Multiple. Nous avons dit que le Multiple dirige l'estimation, c'est-à-dire la conduit, vu qu'il est principe seulement de la tendance, l'entendu étant déterminé par l'Un. Cependant, il ne serait pas trop difficile de démontrer que dans le domaine du réel, si on met l'accent sur l'objet de la relation déterminative sujet-objet qui donne ce domaine, la décision finale concernant le sens et la valeur devra appartenir au Multiple, et non pas à l'Un.

A vrai dire, l'apport de l'Un dans l'évaluation synétique de la réalité a un double aspect. D'une part, il y a le mouvement d'unification de la conscience, sans lequel - comme nous l'avons déjà montré - aucune pensée cohérente ne serait possible. D'autre part, chaque attribut de l'Un établit un niveau d'entendre les quatre attributs du Multiple. Certes, nous ne devons pas oublier que les attributs (ceux de l'Un, comme ceux du Multiple) sont ordonnés par implication. Aussi, quand nous parlons, par exemple, de l'universalité, ou de la multilatéralité, nous entendons eo ipso tous les autres attributs de la série respective. Donc, le plus bas niveau d'entendre est défini par l'unité. Cela veut dire qu'à ce niveau l'être est considéré simplement comme un tout composé ; partant, c'est le niveau des organismes inférieurs. Quant à l'évaluation effective, elle aura lieu suivant les attributs du Multiple. Et ce mode d'évaluer sera répété à tous les niveaux. 
Le second niveau est celui de l'univocité (qui implique aussi l'unité), c'est-à-dire l'échelon sur lequel se trouve tout être ordonné en vue d'un certain dessein. On sait que des êtres relativement inférieurs peuvent atteindre un haut degré de vie organisée (par ex. les abeilles, les fourmis, etc.). Toutefois, leur pouvoir d'organisation est purement instinctif et ne laisse à la liberté qu'une marge infinitésimale. Aussi, leur puissance de signification est-elle très réduite. Cette puissance ne s'affirme intégralement qu'au niveau suivant : celui de l'unicité. C'est, en effet, à partir de ce niveau que nous pouvons parler d'autonomie ou puissance propre de signifier, c'est-à-dire de se justifier soi-même (selon nos définitions du paragraphe 2). Enfin, il est évident que la capacité de signifier est encore approfondie au dernier niveau, celui de l'universalité, car la signification s'avère maintenant capable de représenter un archétype, et partant d'être prise pour modèle. Ainsi, les attributs de l'Un forment une échelle de compréhension du réel, et chaque échelon est pourvu de quatre degrés de valeur, établis par les attributs du Multiple. Nous ne pouvons pas entrer ici dans les détails d'une telle évaluation; cela nous entraînerait trop loin, et nous écarterait du strict sujet de cet article.

\section{IMPLICATIONS ÉPISTÉMOLOGIQUES}

Essayons maintenant de considérer les implications cognitives de la théorie synétique de la valeur. En exprimant les relations épistémologiques, un fait significatif apparaît digne d'être remarqué : la valeur de la connaissance se révèle grâce à deux activités opposées. D’une part, cette valeur dépend de la puissance avec laquelle le sujet connaissant accomplit l'unité et l'univocité des actes de la conscience, donc de la concentration de cette conscience pour constituer une activité cohérente, dans laquelle toutes ses tendances convergent vers l'objet à connaître. D'autre part, la valeur de la connaissance est en fonction de la diversité multiforme de l'objet, ce dernier pouvant se manifester sous une multitude d'aspects, due à la multiplicité indéfinie de modes d'être réel. On remarque aisément quel est le domaine de I'Un et quel est celui du Multiple, et pareillement on comprend dans quel sens nous devons chercher la croissance en valeur. Les domaines respectifs, ainsi que les sens valoriquement croissants, 
sont les mêmes que ceux qui furent constatés jusqu'ici dans l'étude de la théorie synétique de la valeur.

D'ailleurs, la signification profonde de l'acte d'unification de la conscience (le «Je pense») ne pouvait échapper au regard critique de Kant, qui l'a développé à sa manière dans la théorie sur l'aperception transcendantale de la «Critique de la Raison Pure ». On observe, également, chez le philosophe de Königsberg, maintes références à la nature multiple de la donnée sensible (empirique) de la réalité, sans que toutefois il fasse des considérations valoriques, autant en ce qui regarde la puissance de synthèse unificatrice, qu'en ce qui concerne la multiplicité réelle. Mais la synthèse cognitive du «Je pense», sans laquelle tout acte de jugement épistémologique est impossible, présente d'autant plus d'intérêt que nous sommes à même de comprendre que la valeur de cette cogitation se trouve à l'intersection de l'Un et du Multiple, car elle est la résultante des deux mouvements inverses, mais directement proportionnels, d'unification et de multiplication. Il est évident, en effet, que toute connaissance devient d'autant plus valeureuse qu'elle concentre, unit et inclut une diversité plus multilatérale d'entités réelles, c'est-à-dire une multitude d'êtres naturels plus variés et plus étendus dans toutes les directions.

Quant au «Cogito, ergo sum» cartésien, il démontre que l'on ne peut avoir conscience du sum en partant de la multiplicité réelle, mais seulement de l'unité idéale de la conscience, que l' «Ego cogito» pré-suppose. Si nous interprétons l'ergo valoriquement, nous comprenons alors que l'unité, et aussi l'unicité du sum, c'est-à-dire de l'existant naturel, sont impossibles sans l'unité authentique et sans l'unicité véritable du Cogito, qui détermine l'individualité de l'être réel. La puissance d'idéation est, à cet égard aussi, immense: je ne saurais être unique que dans la mesure où «Je pense ». Autrement, c'est-à-dire en étant simplement réel, je ne puis être que singulier, la relation ontologique fondamentale de la réalité se déployant entre singularité et pluralité.

De plus, «Je pense» étant un acte intuitif, donc simple, il opère l'unification de la conscience, et par cette faculté même, soutenant la pensée entière, il rend possible la liberté de la 
conscience. A vrai dire, la majorité des grands philosophes (Descartes, Kant, Husserl, Jaspers) n'ont pas manqué d'être conscients de l'importance et de la signification à valeur de principe de l' «Ego cogito». Ainsi, en tant que puissance de concentration de tous les actes de la conscience, «Ego cogito » a deux implications majeures. La première est directe, notamment la conscience même, laquelle sans cet acte permanent n'aurait pas de cohérence, donc ne pourrait exister. La deuxième implication est indirecte: elle n'est autre que la liberté. En effet, une cogitation dépourvue d'une «fonction convergente» qui lui assure l'univocité, se dissiperait en équivoque et, eo ipso, ne serait plus libre. Se déployant en une multiplicité de sens, une telle cogitation tomberait facilement sous la contrainte des objets dans lesquels elle se laisserait diviser, diversifier, «multiplier». Le divertissement de Pascal n'est-il pas encore à cet égard édifiant? C'est pourquoi, il est nécessaire de maintenir une cohésion perpétuelle, que seul un acte unique - Je pense - peut accomplir, grâce à sa capacité d'être un fondement continu. Donc, il nous est permis de conclure que d'une part la conscience, comme résultante d'une activité autonome (la cogitation), et d'autre part la liberté, comme limite de la même activité, existent uniquement $\in \mathrm{n}$ tant qu'elles présupposent que subsiste un continuum. L'expression de ce continuum révèle la forme stricte de l'Un, donc de la Vérité, par l'acte fondamental Ego cogito.

\section{CONCLUSION}

Ainsi, nous constatons que les implications cognitives du synétisme valorique nous ont ramené de nouveau à l'antique problème de l'Un et du Multiple. Mais alors, si autant la théorie du sens et de la valeur, que celle de la connaissance jugent « dans les limites » de la proportion Un - Multiple, nous nous rendons compte de l'importance philosophique de cette proportion. Il y a, cependant, une condition à remplir si l'on veut que la relation entre l'Un et le Multiple puisse encore présenter de l'intérêt pour la philosophie. En effet, les conclusions auxquelles nous sommes parvenus au cours de l'article impliquent un changement dans la manière de traiter l'antique problème. Ce changement n'est pas difficile à découvrir : le problème n'est plus posé en termes 
ontologiques, mais en termes de sens et de valeur. Cela nous permet de construire une méthode à implications infinies sur les assises de l'Un et du Multiple. C'est la seule condition qui s'impose pour ressusciter de leur sommeil séculaire ces deux idées philosophiques majeures. Mais c'est une «conditio sine qua non».

Bucarest 